\title{
Digit recognition using decimal coding and artificial neural network
}

\author{
Toufik Datsi*, Khalid Aznag, Ahmed El Oirrak \\ Dept. of Computer Science, Cadi Ayyad University, \\ Marrakech, Morocco \\ ${ }^{*}$ Corresponding author: toufik.datsi@ced.uca.ma
}

\begin{abstract}
Current artificial neural network image recognition techniques use all the pixels of an image as input. In this paper, we present an efficient method for handwritten digit recognition that involves extracting the characteristics of a digit image by coding each row of the image as a decimal value, i.e., by transforming the binary representation into a decimal value. This method is called the decimal coding of rows. The set of decimal values calculated from the initial image is arranged as a vector and normalized; these values represent the inputs to the artificial neural network. The approach proposed in this work uses a multilayer perceptron neural network for the classification, recognition, and prediction of handwritten digits from 0 to 9 . In this study, a dataset of 1797 samples were obtained from a digit database imported from the Scikit-learn library. Backpropagation was used as a learning algorithm to train the multilayer perceptron neural network. The results show that the proposed approach achieves better performance than two other schemes in terms of recognition accuracy and execution time.
\end{abstract}

Keywords: Artificial neural network; backpropagation; classification; digit dataset; handwritten digit.

\section{Introduction}

As an important application of pattern recognition theory and computer vision, image recognition has become an interesting research topic in the field of image processing and machine learning (Omarov \& Im Cho, 2017). Face recognition, signature verification, text recognition, face detection, and object detection are all typical examples (Zafar et al., 2019; Tsai et al., 2018; Cho et al., 2018; Al-Hmouz, 2020). Recently, machine learning has been gaining importance in the field of pattern recognition, mainly stimulated by the evolution of artificial neural network models, and particularly the application of multilayer perceptron and convolutional neural networks (CNNs) to various vision-related tasks (Dhillon \& Verma, 2019; Saeed, 2015). Several research studies of pattern recognition with artificial neural networks (ANNs) have been devoted to the recognition or classification of object categories and digit recognition (Girshick et al., 2014; Krizhevsky et al., 2012; Pang \& Yang, 2016). Handwritten digit recognition (HDR) is a major area of machine learning within the field of optical character recognition (OCR) and can be used to solve complex problems (Ali et al., 2019; Alonso-Weber 
et al., 2013). As an interesting problem, this has attracted much attention from research teams (Choi et al., 2019; Damak et al., 2020; Zhu et al., 2018). HDR has been utilized in many applications, for example in banks for reading checks, and in post offices for sorting mail (Tuba et al., 2019). In general, HDR can be divided into the two categories of offline and online techniques (Qinghui et al., 2010; Tagougui et al., 2013). In recent years, the ANN has become the most commonly used tool in machine learning, and its effectiveness has been proven in many applications such as pattern recognition, speech recognition, face recognition, analysis, and image compression (Baptista \& Morgado-Dias, 2013). It has become more competitive with other models used in regression and statistics in terms of its usefulness. An ANN contains a set of different types of neural networks, such as single layer perceptrons, feedforward, networks, multilayer perceptrons, recurrent neural networks, and CNN's (Datsi et al., 2019; Ramzan et al., 2018). It stimulates the biological function of nervous systems and can optimize complex systems that are difficult to model using other techniques such as mathematical modeling (Abiodun et al., 2018). An ANN is made up of a set of neurons interconnected by weights, whose values influence the behavior of the entire structure (Manickam et al., 2017). Learning in neural networks consists of calculating the parameters in such a way that the outputs of the neural network are as close as possible to the desired outputs for the particular examples used in learning. Various techniques can be used to build a neural network model, and the most common in machine learning is called classification. Classification plays an important role in many areas such as image processing (Ghosh et al., 2020), and has become one of the key components of object recognition and handwritten digit recognition (Rashid et al., 2019).

Most feature extraction techniques for digit recognition use all the pixels of an image as the input to the neural network (Alom et al., 2017). Several challenges arise when using this technique, which is related to the large sizes of images, the number of hidden layers in the neural network, and the processing time required.

An efficient feature extraction method for digit recognition is presented in this paper. The proposed method is divided into three parts: at the first stage, we binarized the image using a binarization technique; in the second, each row of the image is encoded as a decimal value; in the third, all decimal values are arranged into a single vector, and a normalization-scaling technique is applied. This vector represents the input to the multilayer perceptron neural network.

The remainder of the paper is organized as follows. Section 2 gives an overview of some previous works in the literature. In Section 3, we describe the proposed methodology, and in Section 4, we present some results and a discussion of the performance of the proposed method. The study is concluded in Section 5.

\section{Related work}

Several methods in the literature have been proposed for digit recognition, and different types of neural networks have been used for this purpose. The multilayer perceptron has been successfully employed with a gradient descent backpropagation algorithm to predict and 
recognize the handwritten digit (Saeed, 2015). A novel method for handwritten digit recognition that was based on a support vector machine (SVM) classifier optimized by a swarm-intelligence guided fireworks algorithm is presented (Tuba et al., 2019). This method used a simple feature set as input for the SVM classifier and the swarm-intelligence-guided fireworks algorithm for the optimization of the parameter tuning of SVM. Another model based on CNN was applied to classify digits using various epochs and numbers of hidden layers (Siddique et al., 2019). The model was trained using the backpropagation algorithm and tested using the MNIST database. Two extraction techniques have been implemented to address the handwritten digit recognition problem (Boukharouba \& Bennia, 2017): the first used a chain code histogram to specify the boundary of the image, and the second used the transition information of the image of the digit in the horizontal and vertical directions. The system was tested using a handwritten sample from the Persian numerals dataset. Another new classification technique based on a multilayer perceptron, which mapped different sets of input data onto the MLP output neurons, is proposed (Keshta, 2017). The Pen-Based Recognition of Handwritten Digits dataset was used to evaluate the model. Based on layering approaches, a minimal convolutional neural network that contained two networks has been proposed for the digit recognition system (Teow, 2017). The first network was responsible for learning the internal representation of the input image, while the second network was responsible for transforming the representation input image to image recognition using successive layers. The mini-batch gradient descent algorithm was used to train the minimal convolutional network model, and the MNIST dataset was used to evaluate the performance of the system. Two classifiers have been used to recognize the handwritten digit (Yu et al., 2015). The LeNet convolutional neural network and support vector machine. The LeNet5 CNN was used as a feature extractor, while the SVM classifier was used as predictor output. The stochastic diagonal Levenberg-Marquardt algorithm was used to train the network, and the method was implemented using the MNIST dataset. A novel technique based on a support vector machine for the classification of digits is implemented (Ingal et al., 2019). The process of the proposed technique is to binarized an image, segmented and extracted the numbers, and recognized them using template matching. The Scikit-learn digit dataset was used to evaluate the system. An optimization method using a neural network was investigated for digit recognition (Yasuoka et al., 2016). Backpropagation, practical swarm optimization (PSO), and random practical swarm optimization (RPSO) were used to achieve high discrimination rates and learning performance. The results show that RPSO worked better and was not dependent on the number of layers in the neural network. The simulation using the RPSO with a three-layer neural network achieved an accuracy of 95\%. A powerful model based on CNN and a support vector machine for the recognition of handwritten digits are developed (Ahlawat \& Choudhary, 2020). In the proposed model, $\mathrm{CNN}$ was worked as an automatic feature extractor and the support vector machine was worked as a binary classifier. The proposed system achieves better recognition over MNIST handwritten digits' dataset. 


\section{Proposed methodology}

\subsection{Artificial neural network}

Several problems involving classification and recognition in different fields of image processing have been solved using ANNs (Wang et al., 2015; Abiodun et al., 2018). An ANN consists of a set of simple elements called neurons or perceptrons (Haripriya \& Jabbar, 2018). An ANNs with different architectures are used for pattern recognition, and the most popular is a multilayer perceptron. In this work, we use both decimal coding and a multilayer perceptron for the classification and recognition of handwritten digits. The digit recognition scenario addressed with the proposed neural network is illustrated in Figure 1.

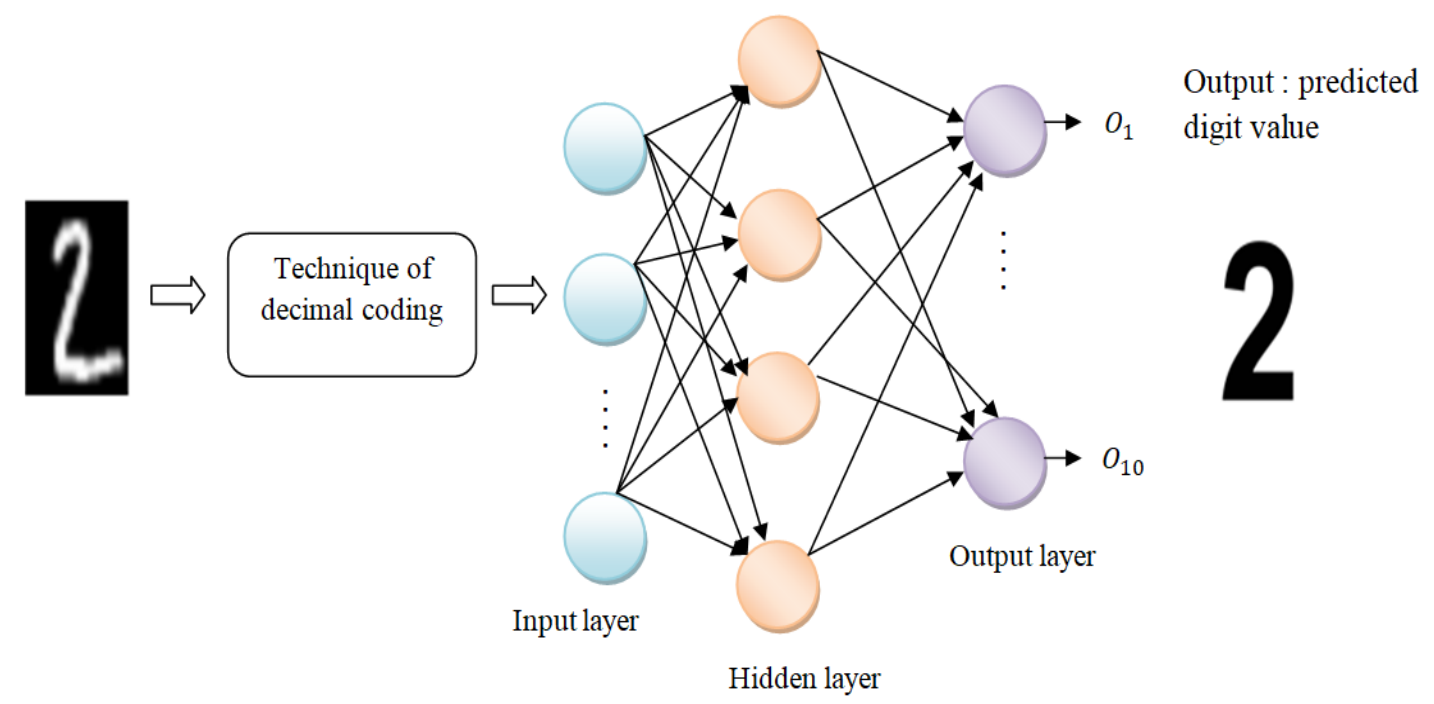

Fig. 1. Digit recognition scenario addressed with the proposed neural network.

The neural network shown above is composed of an input layer that receives the data extracted from the digit image and an output layer that makes a prediction or decision on the input. The layer between the input and output layer is called the hidden layer.

\subsection{Proposed model}

Classification and pattern recognition are the problems most frequently optimized using ANNs. To improve the recognition of handwritten digits and make good predictions, architectures based on a neural network can be used. A multilayer perceptron can ensure high recognition and accuracy at the same time robust training. In this study, we propose a model that uses row decimal coding as a feature extractor and a multilayer perceptron as the architecture of our neural network for the classification and recognition of digits. The proposed model is shown in Figure 2 . 


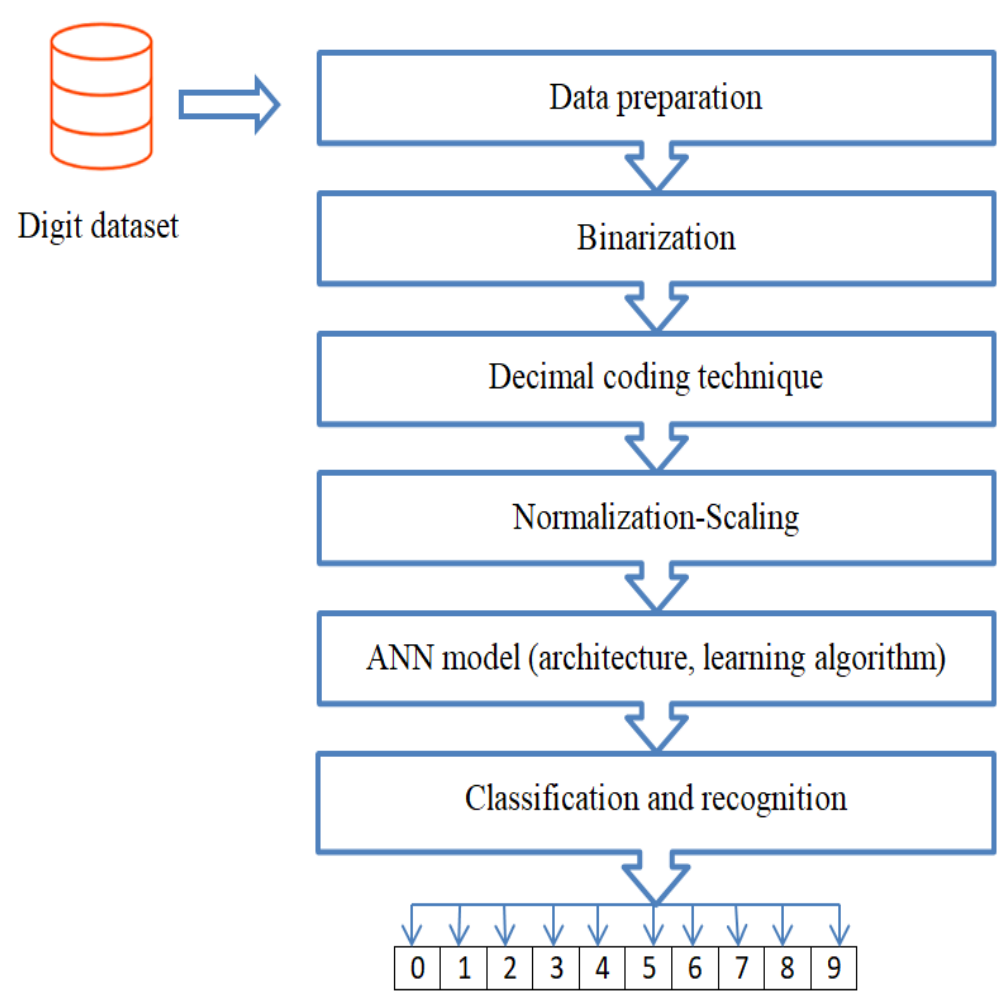

Fig. 2. The proposed model.

\subsubsection{Data preparation}

In this phase, we loaded the digit dataset from the Scikit-learn library, in which each pixel of each image can take a value from zero to 255 . The representation of each image is a matrix with dimensions $8 \times 8$. The image is transformed into a matrix whose elements correspond to individual pixels. After loading the dataset, we created the feature and target arrays, and then split the dataset of 1797 images into two sets: 1432 images for use in training and 365 for use in testing.

\subsubsection{Binarization}

This is a widely used technique in image processing (Mustafa et al., 2018). The process of binarization consists of separating pixels' values into two classes, where black represents the background (a black pixel with minimum of intensity, i.e., equal to zero) and white represents the foreground (a white pixel with maximum intensity, i.e., equal to one). The goal of binarization is to compare the value of each pixel in the image with a fixed threshold T. Given an image I with pixels denoted as $x(i, j)$ and a threshold value $T$, a binarized image $I_{b}$ with $x_{b}(i, j)$ elements is obtained as follows:

$$
\left\{\begin{array}{cc}
\text { if } x(i, j)>=T, & x_{b}(i, j)=1 \\
\text { Else }, & x_{b}(i, j)=0
\end{array}\right.
$$




\subsubsection{Decimal coding technique}

This is an important process in digit recognition. After binarizing the data, each pixel in the image contains zero or one, and the format of the image becomes binary. The next process involves coding each row of the image based on its value; this transformation is called the passage of the binary representation in decimal value. A set of decimal values representing the rows of the digit image are regrouped into a single vector. This vector represents the feature input of the neural network. Figure 3 shows the process of decimal coding. The objective of the process is to reduce the number of pixels in the image given to the neural network.

\begin{tabular}{l}
\multicolumn{6}{|c|}{ Binary values of the image } \\
$2^{7}$ \\
\begin{tabular}{|l|l|l|l|l|l|l|l|}
\hline 0 & 0 & 1 & 0 & 0 & 1 & 0 & 0 \\
\hline 0 & 1 & 0 & 0 & 0 & 0 & 1 & 1 \\
\hline 0 & 0 & 0 & 1 & 1 & 0 & 0 & 0 \\
\hline 1 & 0 & 0 & 0 & 0 & 0 & 0 & 1 \\
\hline 0 & 0 & 0 & 0 & 0 & 0 & 1 & 0 \\
\hline 0 & 0 & 1 & 0 & 0 & 0 & 0 & 0 \\
\hline 0 & 0 & 0 & 0 & 0 & 1 & 1 & 0 \\
\hline 0 & 0 & 0 & 0 & 0 & 0 & 1 & 1 \\
\hline
\end{tabular}
\end{tabular}
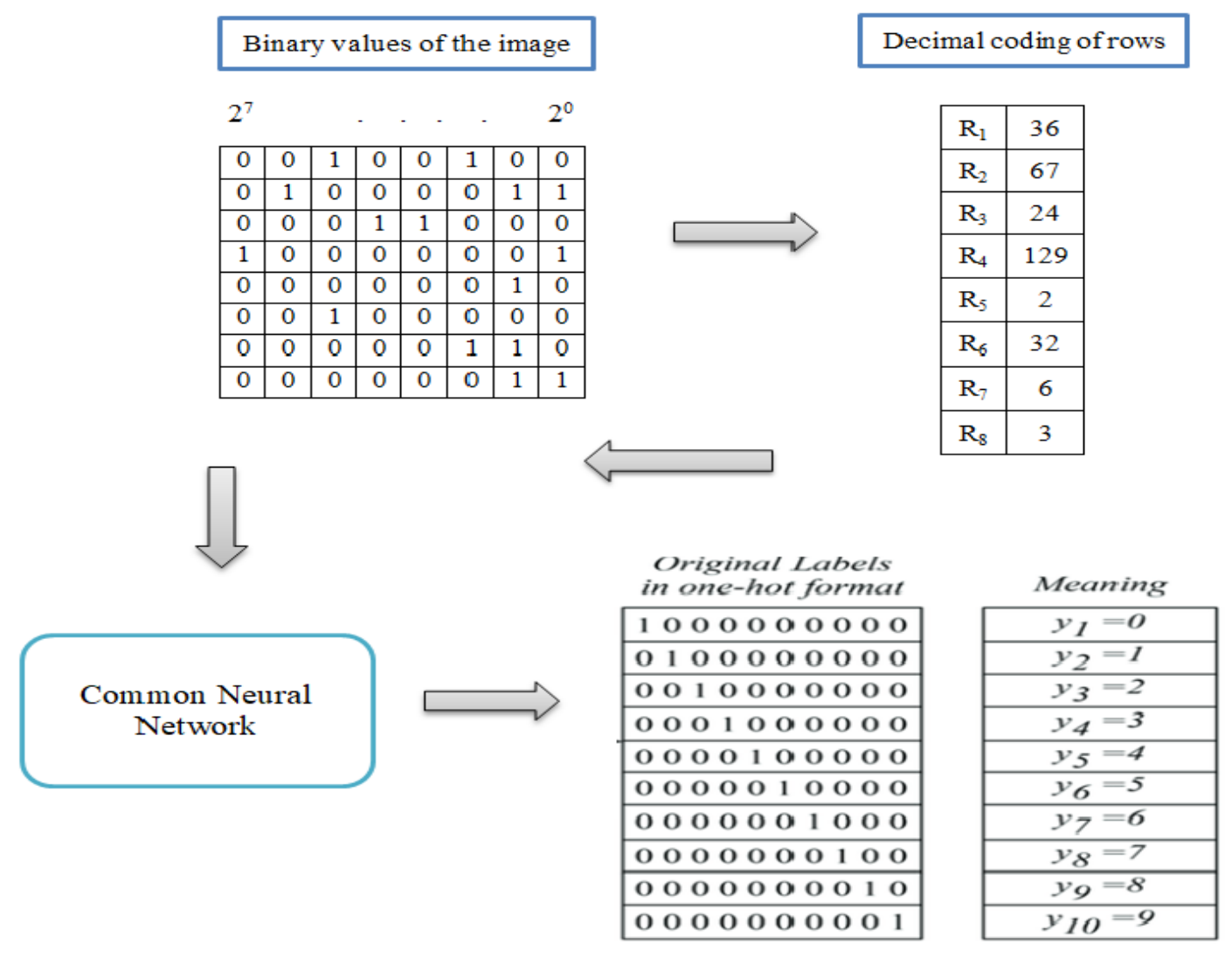

Fig. 3. The process of decimal coding.

\subsubsection{Normalization-Scaling}

This is a technique that is used to normalize data (Saranya \& Manikandan, 2013). In our scheme, the pixels must be scaled before the neural network is trained. In the proposed model, the pixel values are scaled to the range 0 to 1 . The calculation is done using the following equation:

$$
\mathrm{h}_{\mathrm{i}}=\frac{\left(\mathrm{h}_{\mathrm{i}}-\mathrm{C}_{\min }\right)}{\left(\mathrm{C}_{\max }-\mathrm{C}_{\min }\right)}
$$


where $C_{\min }$ is the minimum of the value calculated for each column $C_{\max }$ is the maximum value that calculated for each column, and $h_{i}$ is the data item present in $i^{\text {th }}$ the row.

\subsubsection{ANN model}

In this section, we describe the architecture of our ANN model. The architecture used here is a multilayer perceptron, and backpropagation is used as the learning algorithm (Srinivas et al., 2012; Karmakar et al., 2014). In general, an MLP (Amato et al., 2017) consists of three layers: an input layer, an output layer, and one or more hidden layers. An MLP network with $K$ output and $n_{h}$ hidden nodes can be expressed as:

$$
\mathrm{y}_{\mathrm{k}}=\sum_{\mathrm{j}=1}^{\mathrm{n}_{\mathrm{h}}} \mathrm{w}_{\mathrm{jk}} \mathrm{G}\left[\sum_{\mathrm{i}=1}^{\mathrm{n}_{\mathrm{i}}} \mathrm{w}_{\mathrm{ij}} \mathrm{x}_{\mathrm{i}}+\mathrm{b}_{\mathrm{j}}\right] ; 1 \leq \mathrm{k} \leq \mathrm{K}
$$

where $W_{i j}$ and $W_{j k}$ represents the connection weights between the input and hidden layers and between the hidden and output layers, respectively. The numbers of nodes in the input and hidden layers are denoted by $n_{i} n_{h}$, and respectively. $x_{i}$ is the input to the $i^{\text {th }}$ perceptron in the input layer, and $b_{j}$ is the bias of the $j^{\text {th }}$ perceptron in the hidden layer. G(.) represents the activation function. In our study, we have used the Relu function (Wang et al., 2020) and softmax function (Zhu et al., 2018) as two activation functions in the hidden layer and the output layer, respectively. Figure 4 illustrates the proposed architecture for the neural network and Figure 5 presents the flowchart for the backpropagation algorithm.

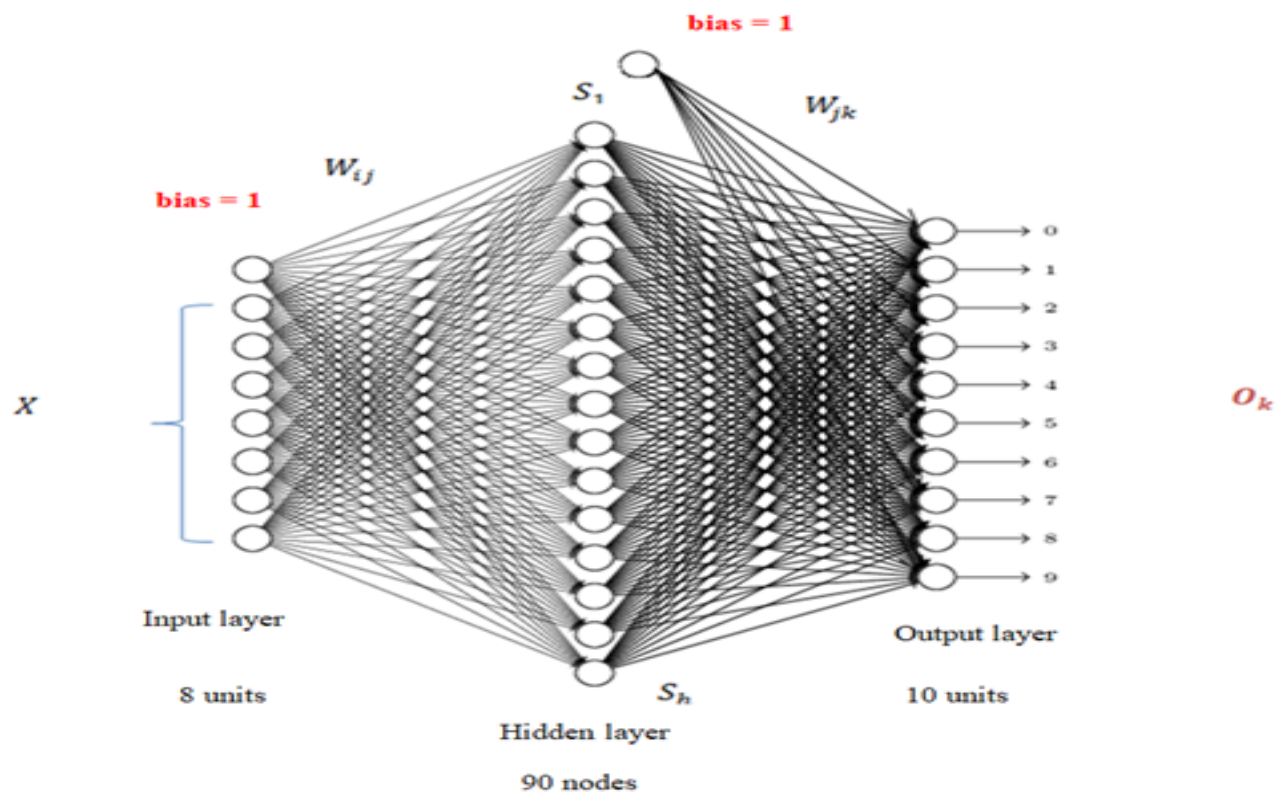

Fig. 4. Proposed architecture for the neural network. 


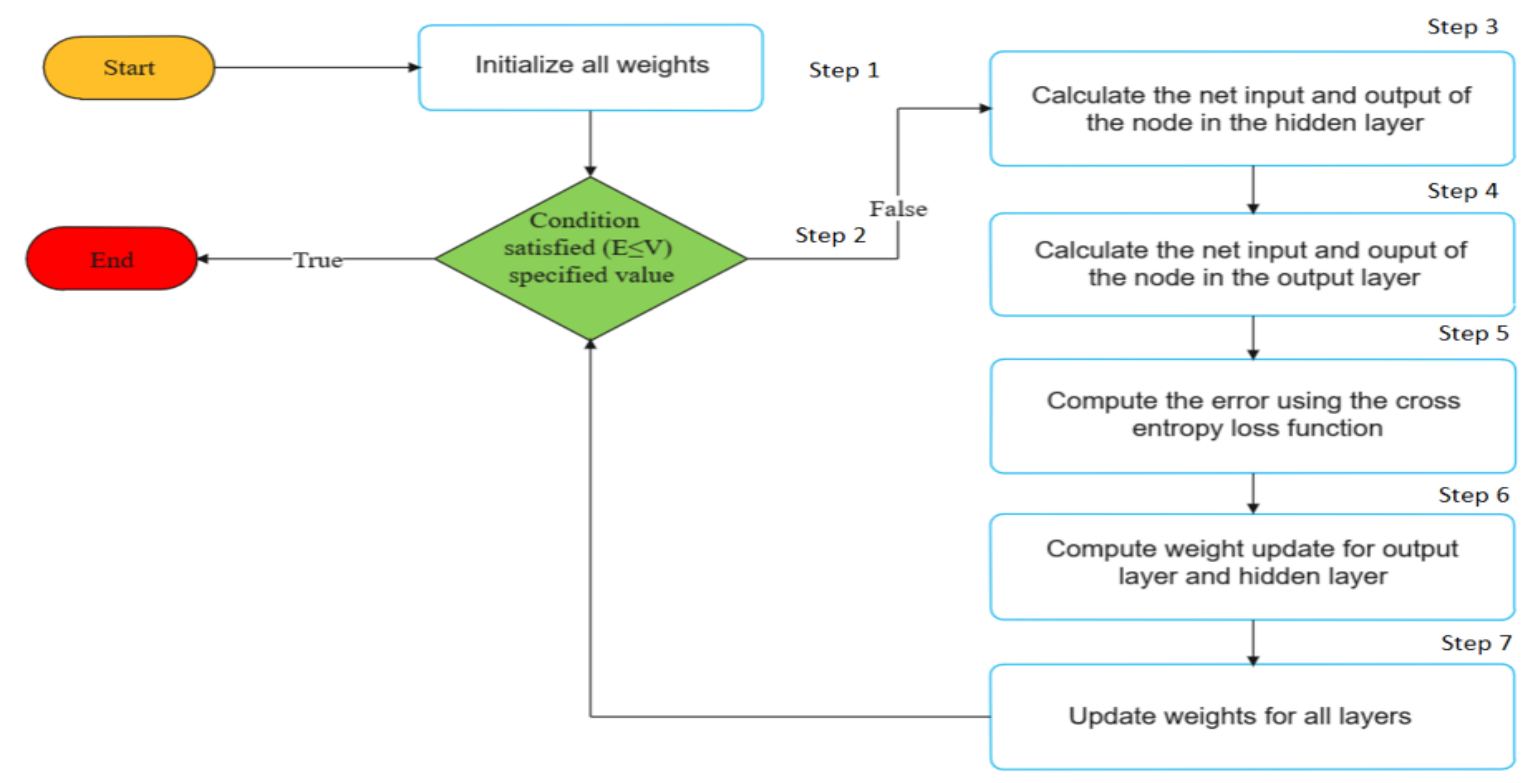

Fig. 5. Flowchart for the backpropagation algorithm.

As shown in Figure 5, the weights of the connections between the layers are initialized with small random values. After initializing the weights, we iterate Steps 3 to 7 as long as the condition is not satisfied. We test the condition in step 2. In this study, the goal of the network was to iterate until $\mathrm{E}<=0.00001$. The function used to minimize the error in the network is the cross-entropy loss function (Bruch et al., 2019).

\subsubsection{Classification and recognition}

After extracting the data from the input image by applying the decimal coding technique and training the network, the last stage is to classify and recognize the handwritten digit. The output layer in the multilayer perceptron consists of 10 perceptrons that recognize the digits from zero to nine. Each digit was represented as $t_{k}$ with the perceptron giving the correct output as one and the remainder as zero. Figure 6 shows the output matrix representing the 10 digits from zero to nine.

$\begin{array}{llllllllll}1 & 0 & 0 & 0 & 0 & 0 & 0 & 0 & 0 & 0 \\ 0 & 1 & 0 & 0 & 0 & 0 & 0 & 0 & 0 & 0 \\ 0 & 0 & 1 & 0 & 0 & 0 & 0 & 0 & 0 & 0 \\ 0 & 0 & 0 & 1 & 0 & 0 & 0 & 0 & 0 & 0 \\ 0 & 0 & 0 & 0 & 1 & 0 & 0 & 0 & 0 & 0 \\ 0 & 0 & 0 & 0 & 0 & 1 & 0 & 0 & 0 & 0 \\ 0 & 0 & 0 & 0 & 0 & 0 & 1 & 0 & 0 & 0 \\ 0 & 0 & 0 & 0 & 0 & 0 & 0 & 1 & 0 & 0 \\ 0 & 0 & 0 & 0 & 0 & 0 & 0 & 0 & 1 & 0 \\ 0 & 0 & 0 & 0 & 0 & 0 & 0 & 0 & 0 & 1 \\ t_{k}^{1} & t_{k}^{2} & t_{k}^{3} & t_{k}^{4} & t_{k}^{5} & t_{k}^{6} & t_{k}^{7} & t_{k}^{8} & t_{k}^{9} & t_{k}^{10}\end{array}$

Fig. 6. The output matrix. 


\section{Results and discussion}

\subsection{Digit dataset}

The digits dataset is a popular and well-known database for the optical recognition of handwritten digits (Karic \& Martinovic, 2013). This dataset was imported from a Python library called Scikit- learn and contained 1797 images, 1797 labels, and 256 levels grey. Each digit image has $8 \times 8=6$ pixels and is labeled with the corresponding category, i.e., the actual digit from 0 to 9 for a total of 10 different labels. A sample image from the digit dataset is shown in Figure 7.

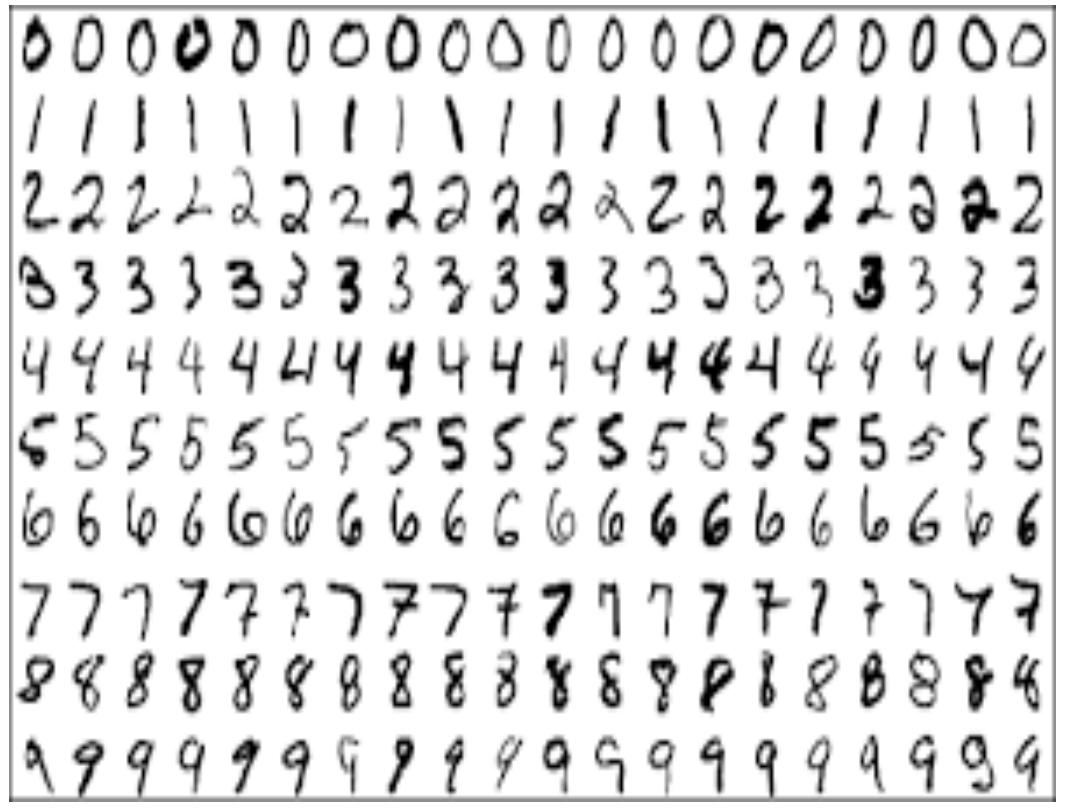

Fig. 7. Sample image from the digit dataset.

The performance of the proposed approach for handwritten digit recognition was evaluated experimentally using 1432 random test samples. The experiments were implemented on the Anaconda platform using Python in a Windows 10 environment on an Intel Core $172.20 \mathrm{GHz}$ with 12 GB RAM. Our approach was tested using the database optical recognition of handwritten digits. The Scikit-learn, Numpy, and matplotlib are the libraries used to implement the proposed model and visualize the digits. A random 1432 samples from the input dataset were used for training, and the 365 samples were used to validate the overall accuracy of the system.

To test the effectiveness of our method, 100 random samples of the 365 validation samples were tested for various numbers of iterations. The results of predictions of handwritten digits are shown in Figures 8, 9, and 10, where incorrect predictions are marked with a red cross. We observed that at iteration 100, three digits were predicted incorrectly, at iteration 250, two digits were predicted incorrectly, and at iteration 400, all digits were predicted correctly. 


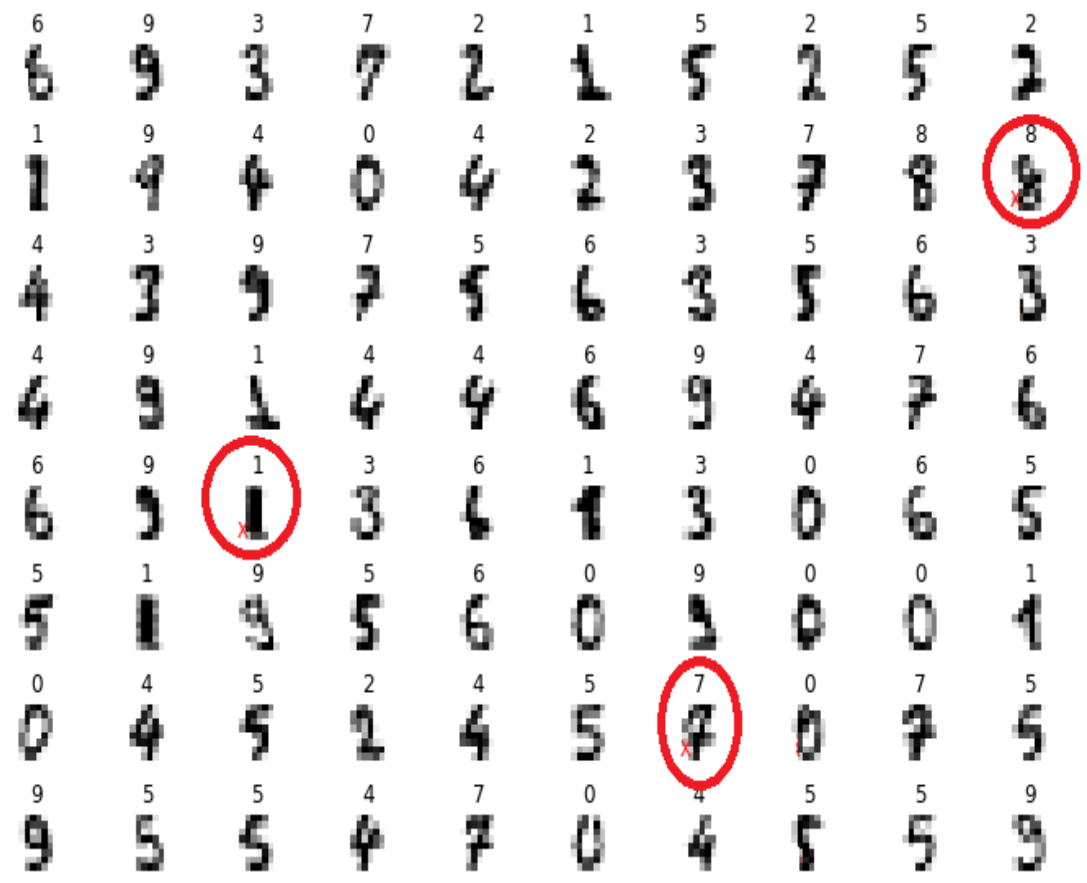

Fig. 8. Testing of 100 samples for 100 iterations.

$\begin{array}{llllllllll}5 & 1 & 7 & 2 & 6 & 2 & 9 & 5 & 3 & 2\end{array}$

$\begin{array}{llllllllll}8 & 2 & 0 & 4 & 1 & 8 & 9 & 3 & 4 & 4\end{array}$

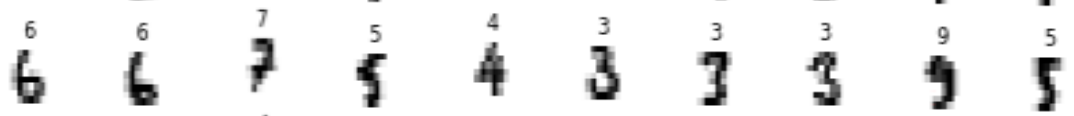

$\begin{array}{llllllllll}7 & 6 & 4 & 4 & 4 & 6 & 9 & 9 & 1 & 4\end{array}$

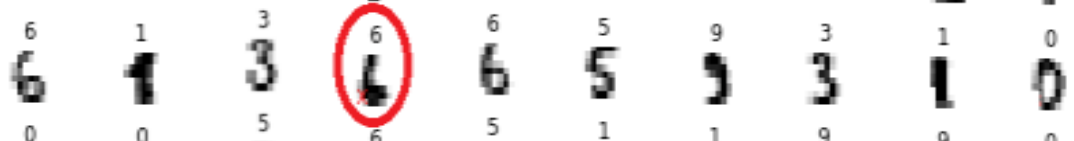

$\begin{array}{llllllllll}0 & 0 & 5 & 6 & 5 & 1 & 1 & 1 & \$ & 9\end{array}$

$\begin{array}{llllllllll}7 & 5 & 2 & 4 & 0 & 5 & 4 & 7 & 5 & 0 \\ 7 & 0 & 0 & 0\end{array}$

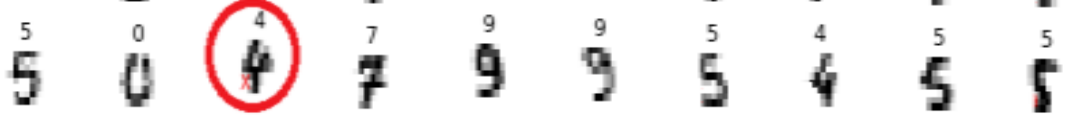

Fig. 9. Testing of 100 samples for 250 iterations. 


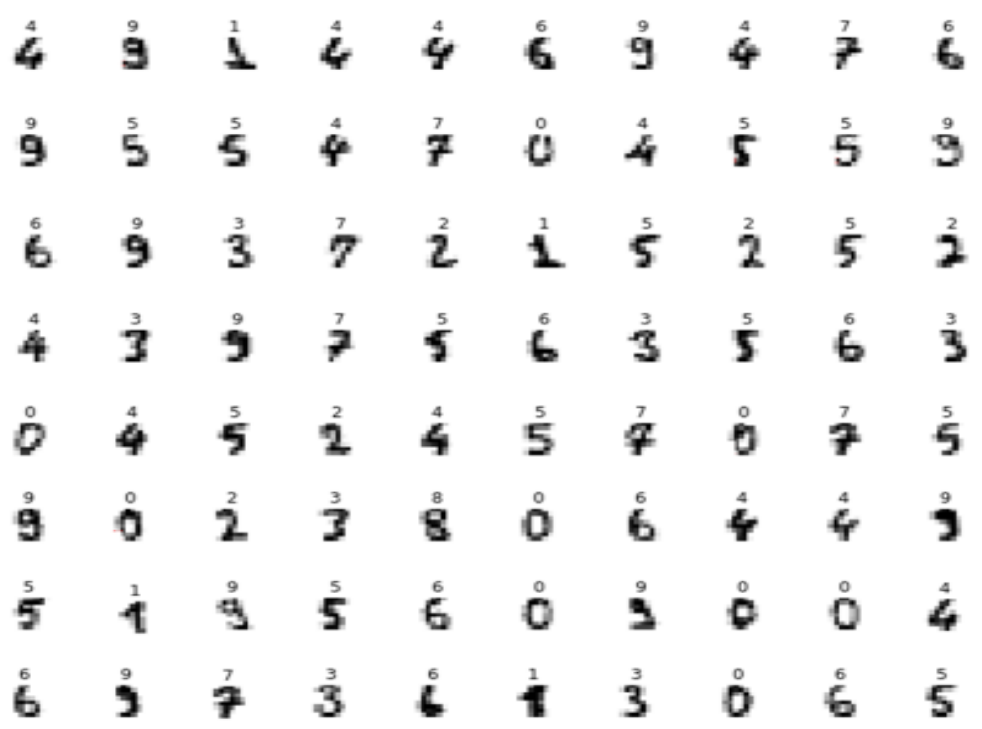

Fig. 10. Testing of 100 samples for 400 iterations.

Table 1 presents a performance analysis based on the number of nodes in the hidden layer and the learning rate. We varied the number of nodes to achieve optimal training of our network. The best accuracy of the system was found for 90 nodes. The learning rate is another hyper parameter used to train the network. Table 2 presents the effect of the learning rate on the error. The best average of error was achieved for a learning rate of 0.01 and iteration of 400 .

Table 1. Performance analysis is based on the number of nodes in the hidden layer.

\begin{tabular}{cc}
\hline Number of nodes in a hidden layer & Accuracy \\
\hline 20 & 93.65 \\
30 & 94.18 \\
40 & 95.45 \\
50 & 96.11 \\
60 & 96.92 \\
70 & 97.65 \\
80 & 98.25 \\
90 & 98.96 \\
\hline
\end{tabular}

Table 2. Effect of learning rate on the error.

\begin{tabular}{ccc}
\hline Learning rate & iteration & Error \\
\hline 0.8 & 50 & 0.005471 \\
0.07 & 100 & 0.005112 \\
0.06 & 150 & 0.004873 \\
0.05 & 200 & 0.004323 \\
0.04 & 250 & 0.003365 \\
0.03 & 300 & 0.003120 \\
0.02 & 350 & 0.002231 \\
0.01 & 400 & 0.001479 \\
\hline
\end{tabular}


To improve the performance of the model for digit recognition, we computed the recognition accuracy of each digit and the execution time. Table 3 presents the recognition accuracy, error rate, and execution time for each digit, and it can be seen that all digits have high recognition accuracy for a low error rate and low execution time.

Table 3. Recognition accuracy and execution time for each digit.

\begin{tabular}{cccc}
\hline Digit & Recognition accuracy (\%) & Error rate & Execution time(s) \\
\hline 0 & 98.35 & 1.65 & 20.25 \\
1 & 97.81 & 2.19 & 22.13 \\
2 & 97.92 & 2.08 & 22.06 \\
3 & 98.79 & 1.21 & 20.05 \\
4 & 97.13 & 2.87 & 22.41 \\
5 & 97.10 & 2.9 & 22.61 \\
6 & 98.15 & 1.85 & 20.45 \\
7 & 97.19 & 2.81 & 22.31 \\
8 & 98.43 & 1.57 & 20.17 \\
9 & 98.90 & 1.1 & 19.81 \\
\hline
\end{tabular}

The accuracy of training and testing was used to prove the effectiveness of the proposed method. The accuracy was calculated using the following equation:

$$
\% \text { Accu }=\frac{\text { NTSPC }}{\text { TNTS }} \times 100
$$

where NTSPC represents the number of testing samples predicted correctly, and TNTS is the total number of testing samples. The results of the accuracy for different numbers of iterations are given in Table 4. As expected, the accuracy increases as the number of iterations increases. At iteration 50, the system had low recognition accuracy, while iteration 400 gave the highest accuracy for the training and testing data.

Table 4. Several iterations vs. accuracy.

\begin{tabular}{ccc}
\hline Iteration & Training & Testing \\
\hline 50 & 94.42 & 94.79 \\
100 & 95.31 & 95.64 \\
150 & 96.12 & 96.52 \\
200 & 96.84 & 96.97 \\
250 & 97.18 & 97.35 \\
300 & 97.79 & 97.95 \\
350 & 98.27 & 98.45 \\
400 & 98.85 & 98.96 \\
\hline
\end{tabular}


A comparison between our proposed method and other approaches is presented in Table 5. In the study by Ingale et al. (2019), where the size of the images was 64 pixels, the accuracy of the model using a training dataset of $75 \%$ of the samples and a test dataset of $25 \%$ was $97.74 \%$. The proposed model achieved better accuracy than the two other methods. Moreover, in our case, the dimensions of the digits were reduced to eight, which helped the system to achieve better training and testing and reduced the recognition time.

Table 5. Comparison between the proposed method and other methods.

\begin{tabular}{lccc}
\hline \multicolumn{1}{c}{ Method } & Dimension & $\begin{array}{l}\text { Training data-test } \\
\text { data }\end{array}$ & Accuracy \\
\hline Ingale et al. (2019) & 64 & $75 \%-25 \%$ & 97.74 \\
Yasuoka et al.(2016) & 64 & $1500-297$ & 95 \\
Proposed method & 8 & $1432-365$ & 98.96 \\
\hline
\end{tabular}

\section{Conclusion and future work}

In this paper, a novel approach based on a decimal coding technique and a neural network has been proposed to recognize handwritten digits. The method involves transforms the initial image of a digit into binary format and then encoding each row of this image as a decimal value. This technique applied is called decimal coding of rows. The set of decimal values are arranged in a single vector and normalized before being used to train the network. The architecture of the neural network used in this study is based on a multilayer perceptron and was tested on a real handwritten digit recognition problem. To reduce the number of pixels in the image, we only implemented decimal coding on the rows represented in the digit image. The advantages of our proposed technique are that we use binarization and pixel reduction, which make it easy to train the model. The proposed neural network was trained and tested on a dataset imported from the Scikit-learn library. Backpropagation is used as the learning algorithm. The results show that the highest accuracy of the proposed system in terms of digit recognition was $98.96 \%$.

In the future, we intend to test our approach with a larger database and will test other feature extraction methods such as local binary patterns, a co-occurrence matrix, and Fourier transforms.

\section{References}

Abiodun, O. I., Jantan, A., Omolara, A. E., Dada, K. V., Mohamed, N. A., \& Arshad, H. (2018). State-of-the-art in artificial neural network applications: A survey. Heliyon, 4(11): e00938.

Ahlawat, S., \& Choudhary, A. (2020). Hybrid CNN-SVM Classifier for Handwritten Digit Recognition. Procedia Computer Science, 167: 2554-2560. 
Al-Hmouz, R. (2020). Deep learning autoencoder approach: Automatic recognition of artistic Arabic calligraphy types. Kuwait Journal of Science, 47(3):2-13.

Ali, S., Shaukat, Z., Azeem, M., Sakhawat, Z., Mahmood, T., \& ur Rehman, K. (2019). An efficient and improved scheme for handwritten digit recognition based on a convolutional neural network. SN Applied Sciences, 1(9): 1-9.

Alom, M. Z., Sidike, P., Taha, T. M., \& Asari, V. K. (2017). Handwritten Bangla digit recognition using deep learning. arXiv preprint :1705.02680.

Alonso-Weber, J. M., Sesmero, M. P., Gutierrez G., Ledezma, A., \& Sanchis, A. (2013). Handwritten digit recognition with pattern transformations and neural network averaging. In International Conference on Artificial Neural Networks, Germany, pp.335-342.

Amato, F., Mazzocca, N., Moscato, F., \& Vivenzio, E. (2017). Multilayer perceptron: an intelligent model for classification and intrusion detection. In 201731 st International Conference on Advanced Information Networking and Applications Workshops (WAINA), Taiwan, pp.686691.

Baptista, D., \& Morgado-Dias, F. (2013). A survey of artificial neural network training tools. Neural Computing and Applications, 23(3): 609-615.

Boukharouba, A., \& Bennia, A. (2017). Novel feature extraction technique for the recognition of handwritten digits. Applied Computing and Informatics, 13(1): 19-26.

Bruch, S., Wang, X., Bendersky, M., \& Najork, M. (2019). An analysis of the softmax cross entropy loss for learning-to-rank with binary relevance. In Proceedings of the 2019 ACM SIGIR International Conference on Theory of Information Retrieval, USA, pp.75-78.

Cho, S. W., Baek, N. R., Kim, M. C., Koo, J. H., Kim, J. H., \& Park, K. R. (2018). Face detection in nighttime images using visible-light camera sensors with a two-step faster region based convolutional neural network. Sensors, 18(9): 1-31.

Choi, E., Chae, S., \& Kim, J. (2019). Machine Learning-Based Fast Banknote Serial Number Recognition Using Knowledge Distillation and Bayesian Optimization. Sensors, 19(19): 1-18.

Damak, T., Kriaa, O., Baccar, A., Ayed, M. B., \& Masmoudi, N. (2020). Automatic Number Plate Recognition System Based on Deep Learning. International Journal of Computer and Information Engineering, 14(3), 86-90.

Datsi, T., El Oirrak, A., \& Aznag, K. (2019). Classification of Iris Plant Using Perceptron Neural Network. In: International Conference on Advanced Intelligent Systems for Sustainable Development, Marrakech, Morocco, pp.176-183. 
Dhillon, A., \& Verma, G. K. (2020). Convolutional neural network: a review of models, methodologies, and applications to object detection. Progress in Artificial Intelligence, 9(2): 85112.

Ghosh, A., Pavate, A., Gholam, V., Shenoy, G., \& Mahadik, S. (2020). Steady Model for Classification of Handwritten Digit Recognition. In Innovation in Electrical Power Engineering, Communication, and Computing Technology, pp.401-412.

Girshick, R., Donahue, J., Darrell, T., \& Malik, J. (2014). Rich feature hierarchies for accurate object detection and semantic segmentation. In Proceedings of the IEEE Conference on Computer Vision and Pattern Recognition, USA, pp.580-587.

Haripriya, L., \& Jabbar, M. A. (2018). A survey on neural networks and their applications. International Journal of Engineering Research in Computer Science and Engineering, 5(4): 6467.

Ingale, K.B., Man, A.S., Swami, V.V. (2019). Handwritten Character Recognition. Journal of Emerging Technologies and Innovative Research, 6(3): 378-381.

Karic, M., \& Martinovic, G. (2013). Improving offline handwritten digit recognition using concavity-based features. International Journal of Computers Communications \& Control, 8(2): 220-234.

Karmakar, S., Shrivastava, G., \& Kowar, M. K. (2014). Impact of learning rate and momentum factor in the performance of the back-propagation neural network to identify internal dynamics of chaotic motion. Kuwait Journal of Science, 41(2):151-174.

Keshta, I. M. (2017). Handwritten digit recognition based on output-independent multi-layer perceptrons. International Journal of Advanced Computer Science and Applications, 8(6):26-31.

Krizhevsky, A., Sutskever, I., \& Hinton, G. E. (2012). ImageNet classification with deep convolutional neural networks. Advances in neural information processing systems, 25: 10971105 .

Manickam, M. V., Mohanapriya, M., Kale, S. A., Uday, M., Kulkarni, P., Khandagale, Y., \& Patil, S. P (2017). Research study on applications of artificial neural networks and E-learning personalization. International Journal of Civil Engineering and Technology, 8(8):1422-1432.

Mustafa, W. A., \& Kader, M. M. M. A. (2018). Binarization of document images: A comprehensive review. Journal of Physics: Conference Series, 1019 (1), pp. 1-9. 
Omarov, B., \& Im Cho, Y. (2017). Machine learning-based pattern recognition and classification framework development. In 2017 17th International Conference on Control, Automation and Systems (ICCAS), South Korea, pp.1-5.

Pang, S., \& Yang, X. (2016). Deep convolutional extreme learning machine and its application in handwritten digit classification. Computational intelligence and neuroscience, 2016: 1-10.

Qinghui, W., Aiping, Y., \& Wenzhan, D. (2010). An improved feature extraction method for individual offline handwritten digit recognition. In 2010 8th World Congress on Intelligent Control and Automation, China, pp.6327-6330.

Ramzan, M., Khan, H. U., Akhtar, W., Zamir, A., Awan, S. M., Ilyas, M., \& Mahmood, A. (2018). A survey on using neural network-based algorithms for handwritten digit recognition. International Journal of Advanced Computer Science and Applications, 9(9):519-528.

Rashid, M., Khan, M. A., Sharif, M., Raza, M., Sarfraz, M. M., \& Afza, F. (2019). Object detection and classification: a joint selection and fusion strategy of deep convolutional neural network and SIFT point features. Multimedia Tools and Applications, 78(12): 15751-15777.

Saeed, A. M. (2015). Intelligent handwritten digit recognition using artificial neural network. International Journal of Engineering Research and Applications, 5 (5): 46-51.

Saranya, C., \& Manikandan, G. (2013). A study on normalization techniques for privacypreserving data mining. International Journal of Engineering and Technology (IJET), 5(3):27012704.

Siddique, F., Sakib, S., Siddique, M., \& Bakr, A. (2019). Recognition of Handwritten Digit using Convolutional Neural Network in Python with Tensorflow and Comparison of Performance for Various Hidden Layers. 2019 5th International Conference on Advances in Electrical Engineering (ICAEE), Bangladesh, pp.541-546.

Srinivas, Y., Raj, A. S., Oliver, D. H., Muthuraj, D., \& Chandrasekar, N. (2012). A robust behavior of Feed Forward Backpropagation algorithm of Artificial Neural Networks in the application of vertical electrical sounding data inversion. Geoscience Frontiers, 3(5): 729-736.

Tagougui, N., Kherallah, M., \& Alimi, A. M. (2013). Online Arabic handwriting recognition: a survey. International Journal on Document Analysis and Recognition (IJDAR), 16(3): 209-226.

Teow, M. Y. (2017). A minimal convolutional neural network for handwritten digit recognition. In 2017 7th IEEE International Conference on System Engineering and Technology (ICSET), Malaysia, pp.171-176. 
Tuba, E., Hrosik, R. C., Alihodzic, A., Jovanovic, R., \& Tuba, M. (2019). Support Vector Machine Optimized by Fireworks Algorithm for Handwritten Digit. In International Conference on Modelling and Development of Intelligent Systems, Romania, pp.187-199.

Tsai, C. C., Tseng, C. K., Tang, H. C., \& Guo, J. I. (2018). Vehicle detection and classification based on deep neural networks for intelligent transportation applications. In 2018 Asia-Pacific Signal and Information Processing Association Annual Summit and Conference (APSIPA ASC), USA, pp.1605-1608.

Wang, L., Zeng, Y., \& Chen, T. (2015). Backpropagation neural network with adaptive differential evolution algorithm for time series forecasting. Expert Systems with Applications, 42(2): 855-863.

Wang, Y., Li, Y., Song, Y., \& Rong, X. (2020). The Influence of the Activation Function in a Convolution Neural Network Model of Facial Expression Recognition. Applied Sciences, 10(5): 1897.

Yasuoka, Y.; Shinomiya, Y.; Hoshino, Y. (2016). Evaluation of optimization methods for a neural network. Proceedings of the 8th International Conference on Soft Computing and Intelligent Systems (SCIS) and the $7^{\text {th }}$ International Symposium on advanced Intelligent Systems (ISIS), Sapporo, Japan, pp.92-96.

Yu, N., Jiao, P., \& Zheng, Y. (2015). Handwritten digits recognition base on improved LeNet5. In 27th Chinese Control and Decision Conference (2015 CCDC), China, pp.4871-4875.

Zafar, U., Ghafoor, M., Zia, T., Ahmed, G., Latif, A., Malik, K. R., \& Sharif, A. M. (2019). Face recognition with Bayesian convolutional networks for robust surveillance systems. EURASIP Journal on Image and Video Processing, 2019(1): 10.

Zhu, J., Ma, H., Feng, J., \& Dai, L. (2018). ID card number detection algorithm based on convolutional neural network. In AIP Conference Proceedings. 1955(1), pp. 1-5.

Submitted: $\quad 16 / 04 / 2020$

Revised: $\quad 24 / 01 / 2021$

Accepted: $\quad$ 24/01/2021

DOI: $10.48129 / \mathrm{kjs} . v 49 \mathrm{i} 1.9556$ 